\title{
Visual orientation illusions: Global mechanisms involved in hierarchical effects and frames of reference
}

\author{
ROBERTA DAINI \\ Università degli Studi di Milano-Bicocca, Milan, Italy \\ and IRCCS S. Lucia, Rome, Italy \\ and \\ PETER WENDEROTH and STUART SMITH \\ Macquarie University, Sydney, Australia
}

\begin{abstract}
Five experiments were conducted in order to determine which of two hypotheses, initially proposed by Rock (1990), accounts for interactions between oriented elements in a visual scene. We also explored the suggestion that two hypothetical processes - namely, frame of reference and hierarchical organization describe phenomena arising from distinct mechanisms (Spinelli, Antonucci, Daini, Martelli, \& Zoccolotti, 1999). Double inducing stimulus versions of one-dimensional and two-dimensional tilt illusions, the rod-and-frame illusion, and combinations of these were used. Our data suggest that both hypotheses can predict orientation interactions in conditions in which only one mechanism-namely, the global visual mechanism of symmetry axes extraction (Wenderoth \& Beh, 1977) - is activated. Which hypothesis is appropriate to predict the perceived orientation depends on some physical features of the objects.
\end{abstract}

When a vertical test rod is surrounded by a tilted square inducing frame, it appears tilted, and the same phenomenon can be observed when a vertical test grating is surrounded by a tilted inducing grating. These two effects are called the rod-and-frame illusion (RFI) and the tilt illusion (TI), respectively, and several different theories have been proposed to explain these effects.

Some authors have suggested that both orientation illusions are due to similar mechanisms (Wenderoth, 1997; Wenderoth \& Johnstone, 1988b). If discussion is restricted to relatively small, centrally viewed stimuli, two main mechanisms have been postulated to underlie the illusory effects (Spinelli, Antonucci, Daini, \& Zoccolotti, 1995; Wenderoth \& van der Zwan, 1991). One mechanism refers to lateral inhibitory interactions between orientationselective neurons in the primary visual cortex (Blakemore, Carpenter, \& Georgeson, 1970). This mechanism produces repulsion effects, so that the test stimulus appears rotated away from the inducing orientation (Wenderoth \& Johnstone, 1987, 1988b). These lateral inhibitory interactions

This research was supported in part by an Australian Research Council Large Grant and in part by an MIVR WFIN Grant 2001 to G. Vallar. The first author's visit to Macquarie University was sponsored by a University of Rome Ph.D. scholarship, and the second author's visit to the University of Rome was sponsored partly by Macquarie University's Overseas Study Programme and partly by the University of Rome. Correspondence concerning this article should be addressed to R. Daini, Department of Psychology, Università degli Studi Di Milano-Bicocca, Piazza dell'Ateneo Nuovo 1, 20126 Milano, Italy (e-mail roberta.daini@ unimib.it). can be removed or reduced by inserting a gap between the test and the inducing stimuli (Poom, 2000; Tolhurst \& Thompson, 1975; van der Zwan \& Wenderoth, 1995; Virsu \& Taskinen, 1975; Wallace, 1969; Wenderoth \& Johnstone, 1988b; Zoccolotti, Antonucci, \& Spinelli, 1993).

The second mechanism refers to the influence of the global inducer orientation on the perception of the test orientation, based on the extraction of symmetry axes (Wenderoth \& Beh, 1977). When test and inducing stimuli abut, large repulsion effects occur at small inducer tilts $\left(10^{\circ}-20^{\circ}\right)$, and smaller attraction effects occur at larger tilts (RFI, $30^{\circ}-40^{\circ}$; $\mathrm{TI}, 70^{\circ}-80^{\circ}$ ). However, when lateral inhibition effects are removed by inserting a gap between the inducer and the test stimuli, smaller equal and opposite repulsion and attraction effects remain. These residual global effects are thought to be activated by the extraction of figural symmetry axes, so that the repulsion effects are due to real axes parallel to the real contours of the inducer, whereas the attraction effects are due to virtual axes. These are either the diagonals of square frames or plaids or the orientation orthogonal to a one-dimensional (1-D) inducing grating (see van der Zwan \& Wenderoth, 1995, and Wenderoth, 1997, for summaries).

It should be noted that when observers view large, peripheral RFI stimuli, presented in a dark room, a third mechanism has been identified. It refers to the visuovestibular interactions shown by ocular torsion and sensations of self-tilting (Ebenholtz \& Benzschawel, 1977; Goodenough, Sigman, Oltman, Rosso, \& Mertz, 1979) present either in subjects with their heads tilted or in sub- 
jects with the head upright and looking at a tilted large square frame (Howard, 1982). The influence of this mechanism is removed by turning on the light or using a small centrally viewed display (Spinelli, Antonucci, Daini, Fanzon, \& Zoccolotti, 1995).

Studies on visual orientation illusions are important for understanding how the orientation of a target stimulus is influenced by the orientation of other objects. However, it is even more important to study how the visual system works when more than one inducing stimulus is present, given that in everyday life, many objects are present in the visual field at any one time. With the purpose of clarifying the influence of multiple inducers on the orientation of a test stimulus, we have considered two apparently mutually exclusive hypotheses, which were proposed by Rock (1990) and have been discussed by other authors.

1. Frame of reference. This Gestalt concept refers to the influence of one object on the perception of the characteristics of another object. Rock (1990) used the frame-of-reference concept in the motion and orientation illusion domains to refer to the influence of the most external stimulus on the judgment of features of all included stimuli. According to Rock, the RFI is due to the observer's use of the tilted square frame as a surrogate for the spatial coordinates of the world.

Empirical evidence for this hypothesis was obtained by using an inner vertical square frame added to the standard large, peripheral frame. In that condition, most of the outer frame effect was still present (DiLorenzo \& Rock, 1982). Spinelli, Antonucci, Daini, Fanzon, and Zoccolotti (1995) replicated those data and suggested that the frame-of-reference effect could be due to visuovestibular mechanisms. Ebenholtz and Utrie (1982, 1983; Ebenholtz, 1985) studied the effect of either an inner or an outer circle on the RFI with a large frame in peripheral view. Consistent with the frame-of-reference hypothesis, they found that the outer, but not the inner, circle induced a large reduction in the RFI.

2. Hierarchical organization. According to Rock (1990), when environmental and egocentric coordinates are well defined, the perceptual system should tend to organize units of the field hierarchically, from the outer to the inner stimulus. Each element in the field should be "perceived primarily in terms of its changing relation to its immediate frame of reference rather than to any external frame" (Rock, 1990, p. 255). In support of this notion, Palmer (see Rock, 1990) found that in a double rectangular frame, the direction of pointing of an inner equilateral triangle test stimulus is influenced in a hierarchical way.

Zoccolotti, Antonucci, Daini, Martelli, and Spinelli (1997), using an inner vertical frame added to an RFI display in central view presentation, found that the innermost of the two surrounding square frames entirely determined the perceived orientation of the test rod. However, in this case, the outermost inducing stimulus affected the perceived orientation of the inner frame, which in turn affected the test stimulus. They described this effect in terms of Rock's (1990) hierarchical organization hypothesis and suggested that global mechanisms were involved.

Our aim was to test the difference between hierarchical organization and frame-of-reference effects postulated by Rock (1990) and to test for any connection with global visual mechanisms. We used double inducing stimulus conditions because they are able to differentiate between an effect from the outermost to the innermost stimulus (hierarchical organization) and an effect from the outermost to every stimulus inside it (frame of reference).

Central view presentation and relatively small displays were used to eliminate visuo-vestibular interactions: A gap between the two inducers and a gap between the inner inducer and the test stimulus were used in order to remove lateral inhibitory mechanisms; in these conditions, it is expected that only global visual processing will be active. Moreover, small orientations $\left(10^{\circ}-15^{\circ}\right)$ of the outermost inducer stimulus were used, which induces repulsion effects in the standard condition (one inducer stimulus).

According to Rock's (1990) theory, by using a double inducing stimulus and central view presentation, we should obtain only hierarchical effects. The outermost inducing stimulus would be expected to act only as a frame of reference in conditions in which no other visual information is available, unlike the conditions used here. Finally, according to Zoccolotti et al. (1997), we should observe hierarchical effects in all cases of RFI and TI, as the result of global visual mechanisms.

\section{EXPERIMENT 1}

It was suggested that the hierarchical effect, obtained with a double RFI display, may be due to global orientation processing mechanisms (Zoccolotti et al., 1997), and other studies on visual orientation illusions have assumed that the same global mechanism underlies both the TI and the RFI (e.g., Wenderoth \& van der Zwan, 1991).

According to this view, it might be expected that a double grating TI (see Figure 1) would produce results similar to the double frame RFI-that is, the outer grating annulus should have no immediate effect on the test grating but, rather, would affect it via its effect on the inner grating annulus.

To test this hypothesis, we manipulated the inner inducing grating orientation, keeping the outer grating orientation fixed at $15^{\circ}$ clockwise $(\mathrm{CW})$, at which the peak TI repulsion effect occurs (see Wenderoth, Johnstone, \& van der Zwan, 1989).

The hierarchical organization hypothesis predicts an indirect, counterclockwise (CCW) vertical setting error when the inner inducing grating is tilted $\mathrm{CCW}$ or is vertical. Repulsion effects by the outermost inducing grating toward the inner inducing stimulus should influence the target indirectly, by affecting the perceived orientation of the inner inducing stimulus, which then directly affects the target's perceived orientation.

Alternatively, the frame-of-reference hypothesis predicts a direct, $\mathrm{CW}$ error (repulsion effect) in all three 



Figure 1. The stimulus displays used in the experiments. (A) Double grating inducer and grating test. (B) Double frame inducer and test rod. (C) Double plaid inducer and test grating. (D) Grating-frame inducer and test rod. (E) Frame-grating inducer and test rod.

inner grating conditions, owing to the dominance of the outer inducer.

\section{Method}

Subjects. Twenty student volunteers from Macquarie University, 10 males and 10 females, participated in the study. All had normal or corrected-to-normal vision.

Stimuli and Apparatus. Stimulus displays were presented on a Silicon Graphics D-M2ONC 19-in. color display monitor (frame rate $=120 \mathrm{~Hz}$, interlaced frame rate $=60 \mathrm{~Hz}$ ) interfaced with a Silicon Graphics Indigo 2 workstation. Special purpose software was designed and written to control stimulus presentation conditions during the experiment. During test conditions, a central circular square wave test grating of $1^{\circ}$ diameter was surrounded by two larger, concentric, annular square wave gratings of the same spatial frequency as the test grating $(3 \mathrm{cpd})$ and separated from each other by a gap of $1^{\circ}$ of visual angle. The inner annulus was separated from the test stimulus by a $1^{\circ}$ gap to eliminate lateral inhibitory interactions (Virsu \& Taskinen, 1975; Wallace, 1969; see also Wenderoth \& Johnstone, 1988b). The inner and the outer annuli were $1^{\circ}$ thick. Thus the outside diameter of the inner annulus was $5^{\circ}$, and that of the outer annulus was $9^{\circ}$ (see Figure 1A).

When measured on a low-frequency square wave grating with a Tektronix $\mathrm{J} 161^{\circ}$ digital luminance probe, maximum luminance of the light bars was $62.5 \mathrm{~cd} / \mathrm{m}^{2}$, whereas the luminance of the dark bars was $0.6 \mathrm{~cd} / \mathrm{m}^{2}$. Thus, the Michelson contrast of both the test stimulus and the inducing gratings, defined as $\left[\left(\mathrm{L}_{\max }-\mathrm{L}_{\min }\right) /\left(\mathrm{L}_{\max }\right.\right.$ $\left.+\mathrm{L}_{\text {min }}\right)$ ], was 0.98 . All the stimuli were constructed with Adobe Photoshop.

Orientation adaptation effects were avoided by using a brief $(500 \mathrm{msec})$ stimulus presentation and an interstimulus interval of $3 \mathrm{sec}$

Procedure. The subjects' responses were recorded using the outer pair of buttons on a three-button mouse to indicate whether the central circular grating appeared to be tilted to the left or the right of perceived vertical. The subjects were seated in a darkened laboratory in which all external cues to vertical were removed by attaching to the display monitor a black cardboard $9^{\circ}$-diameter mask. A black cloth was draped over the area between the display and a padded chinrest in which the subjects placed their heads. The chinrest was located $1.14 \mathrm{~m}$ from the display so that $2 \mathrm{~cm}$ on the screen subtended $1^{\circ}$ of visual angle. Each subject was tested under three conditions of inner inducing grating orientation: upright $\left(0^{\circ}\right)$, tilted in same direction and by the same amount as the outer inducer $\left(15^{\circ} \mathrm{CW}\right.$, or $\left.+15^{\circ}\right)$, or tilted in the direction opposite to that of the outer inducer $\left(15^{\circ} \mathrm{CCW}\right.$, or $\left.-15^{\circ}\right)$. Only a single level of outer inducing grating orientation condition was used $\left(+15^{\circ}\right)$. Prior to each set of trials in which both the test stimulus and the inducing fields were presented simultaneously (called the test condition), the subjects were run under a pretest condition in which the test field alone was presented and orientation judgments of it were made. A pretest condition always preceded a test condition to control for possible drift in subjective vertical over the experiment. A double randomly interleaved staircase technique was used (Wetherill \& Levitt, 1965). Each staircase was started from a test stimulus orientation randomly chosen from the range of $\pm 10^{\circ}$ from gravitational vertical. Step size was initially $2^{\circ}$ and was reduced to $1^{\circ}$ after the third reversal. Staircases were then run for 10 more reversals, with the point of subjective vertical (PSV) estimated by averaging the peaks and valleys of the last 6 reversals. The subjects were instructed to be as accurate as possible in their judgments and to maintain fixation on a small dark spot in the center of the display during each trial. Following the 500-msec presentation duration of each trial, the entire screen was blanked to $6.4 \mathrm{~cd} / \mathrm{m}^{2}$, which was sufficient to eradicate any afterimages. After the subject responded, the blank field remained present for $3 \mathrm{sec}$, after which the next trial was initiated. The entire session lasted approximately $30 \mathrm{~min}$.

\section{Results}

All PSVs to the left of vertical were signed negative, and those to the right were signed positive. Magnitude and direction of the orientation illusion was calculated as test PSV minus pretest PSV. The mean illusions and standard errors are shown in Figure 2A. Mean illusions were $-0.99^{\circ}$ when the inner inducer was tilted in the direction opposite to the outer inducer, $+0.32^{\circ}$ in the upright inner inducer condition, and $+1.09^{\circ}$ when the inner inducer was tilted in the same direction as the outer in- 


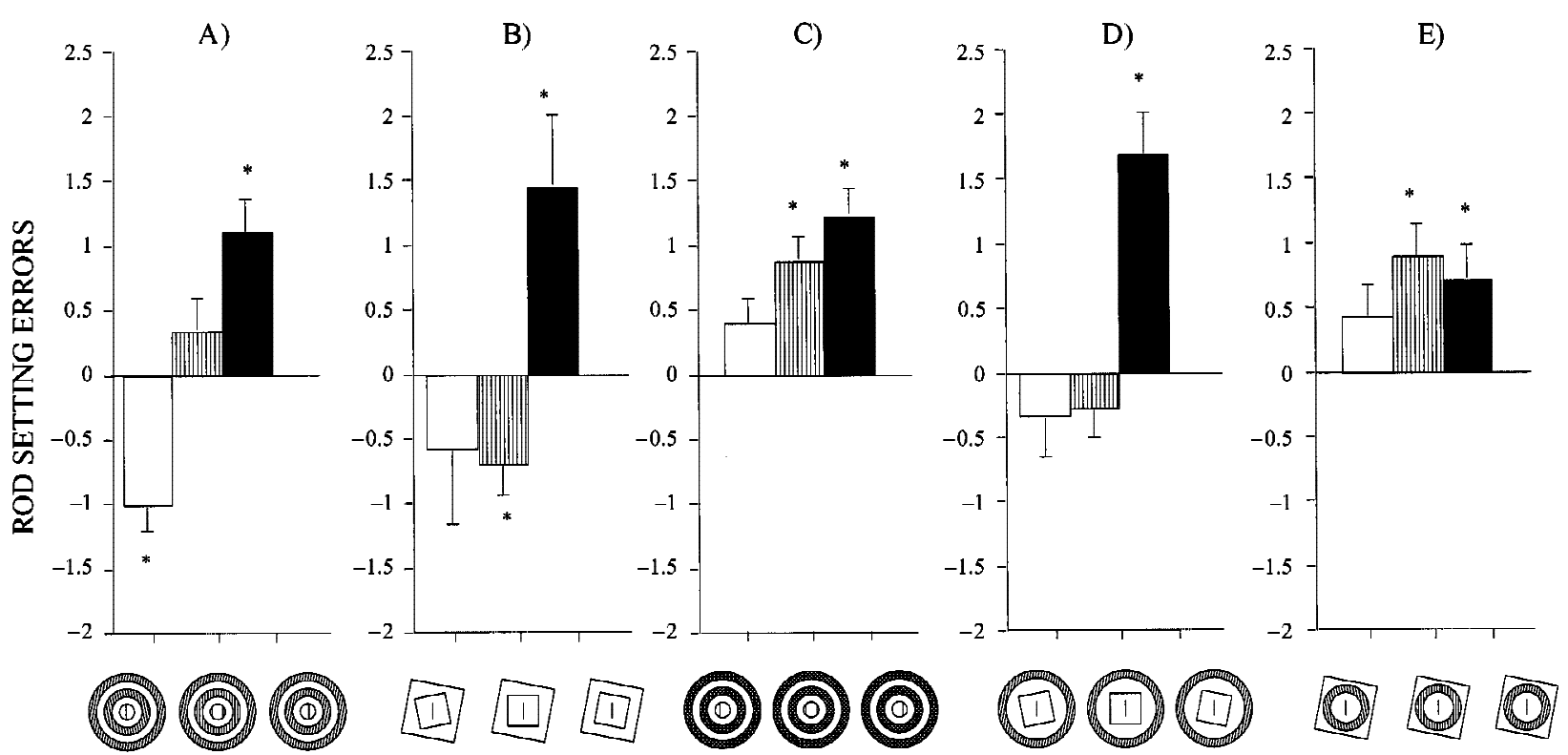

Figure 2. Mean illusions and standard errors obtained in Experiments 1, 2, 4, and 5 are shown in panels A-E, respectively. The insets below the results show the stimulus displays. Outer inducer orientation was fixed $\left(15^{\circ}\right.$ clock wise for the outer grating conditions and $11^{\circ}$ clockwise for the outer square frame and the outer plaid conditions), whereas inner inducer orientation was varied (counterclockwise, in the opposite direction to the outer inducer; $0^{\circ}$; and clockwise, in the same direction as the outer inducer).

ducer. Only the CCW $[t(19)=-4.411, p=.001]$ and $\mathrm{CW}$ $[t(19)=3.798, p<.005]$ inner inducer tilts were significantly different from zero. An analysis of variance (ANOVA) with the orientation condition as the repeated measure showed a significant effect $[F(2,38)=26.59$, $p<.0001]$. A Sheffé post hoc comparison showed that the illusion at each orientation differed from the other two $(p<.05)$.

\section{Discussion}

The results indicate that the illusory effect was due mainly to the internal grating. When the inner inducer was tilted in a CCW direction, the illusion was in the same direction, as would be expected if it was determined only by the inner grating. When the inner inducer was tilted in a CW direction, the effect was in the CW direction. The fact that the two effects were almost identical in magnitude suggests that the outer frame (always tilted CW) had no effect at all.

For the $0^{\circ}$ inner grating condition, there was no significant effect. This confirms the relevance of the inner inducing grating and the ineffectiveness of the outer grating. In conclusion, a double grating TI display acted as if it were a single TI, as if the external grating were absent; neither the hierarchical organization nor the frameof-reference hypothesis can account for this result.

\section{EXPERIMENT 2}

Zoccolotti et al. (1997) obtained a hierarchical organization effect with a double RFI display, using the method of constant stimuli and with stimulus figures printed on paper. Palmer (see Rock, 1990) demonstrated the same effect with a double rectangle frame and an equilateral triangle test stimulus. Experiment 2 was performed to test the effect of a double square frame on the apparent vertical in order to compare this with the TI results of the previous experiment, using an identical apparatus and method. We expected to replicate Zoccolotti et al.'s (1997) results and to obtain a hierarchical effect-thus, a CCW effect for the upright inner frame and the $\mathrm{CW}$ outer frame orientations.

The experiment was conducted in order to demonstrate that the difference between our results with double frame TIs in Experiment 1 and previous results on double frame RFI (Zoccolotti et al., 1997) was due not to apparatus differences, but to physical features of the inducing frames.

\section{Method}

Subjects. Twenty new subjects (10 males and 10 females) were drawn from the same population as those in Experiment 1.

Stimuli and Apparatus. The apparatus was the same as that used for the TI experiment, and the stimuli were constructed with the same software. During the test conditions, a central rod $1^{\circ}$ long and $0.1^{\circ}$ thick was surrounded by two larger, square frames of the same thickness as the test rod. The sides of the inner frame were $3^{\circ}$ long, and the sides of the outer frame were $7^{\circ}$ long. The gaps between the rod and the inner frame and between the inner and the outer frames were never smaller than $1^{\circ}$ (see Figure 1B).

Procedure. The procedure was the same as that in the previous experiment, except that the subjects were required to judge the verticality of a rod rather than a circular test grating. Each subject was tested once again with three inner inducing square orientations: up- 
right, tilted in same direction and by the same amount as the outer square, and tilted in the opposite direction to the outer square. Only a single level of inducing outer square orientation condition was used $\left(11^{\circ} \mathrm{CW}\right)$ where the peak RFI occurs (Antonucci, Fanzon, Spinelli, \& Zoccolotti, 1995). The entire session lasted approximately $40 \mathrm{~min}$.

\section{Results}

The mean illusions and standard errors are shown in Figure $2 \mathrm{~B}$. Mean errors from the vertical were $-0.57^{\circ}$ when the inner frame was tilted in the opposite direction to the outer frame, $-0.68^{\circ}$ in the upright inner frame condition, and $+1.43^{\circ}$ when the inner frame was tilted in the same direction as the outer frame. The means of the upright $[t(19)=-2.48, p<.05]$ and the $0^{\circ}[t(19)=2.42$, $p<.05]$ inner frame conditions were significantly different from zero.

An ANOVA with the orientation condition as the repeated measure showed a significant effect $[F(2,38)=$ $7.55, p<.005]$. A Sheffé post hoc comparison showed that the means at both the $\mathrm{CCW}$ and the $0^{\circ}$ orientations of the inner stimulus were different from that at the $\mathrm{CW}$ orientation ( $p<.01$ and $p=.005$, respectively), but not from each other.

\section{Discussion}

The effects obtained in the double square RFI contrast with the TI results of Experiment 1, suggesting a significant effect of the outer frame on the inner frame. Nevertheless, the PSV was in the same direction as the outer frame tilt only for a congruently tilted inner frame- that is to say, the effect of the outer frame on the test is not direct. In the $0^{\circ}$ inner frame condition, the PSV was tilted in the opposite direction to the outer frame, consistent with the results obtained by Zoccolotti et al. (1997).

A plausible explanation is that in the $0^{\circ}$ inner frame condition, the outer frame induces an illusory effect, but only on the inner frame, and the rod is influenced only by this illusory inner frame orientation.

When the inner inducing frame was tilted in the opposite direction to the outer frame $\left(11^{\circ} \mathrm{CCW}\right)$ the illusory effect on the test stimulus was negative but not significant. This result suggests that the external frame makes the inner frame appear even more tilted than $11^{\circ} \mathrm{CCW}$ and, hence, the effect decreases, $11^{\circ}$ being the frame orientation that induces the peak of the illusory effect.

It might be suggested that the effect shown is, in fact, due to the tendency of observers to perceptually group the inner frame and the rod into a single object and that the effect of the outer frame is to induce a CCW tilt of this grouped "object." However, this cannot be the explanation, because it predicts that the rod will appear tilted CCW and so will be set $C W$ to compensate. This is the opposite of the observed effect: The outer frame makes the inner frame, not the rod, appear tilted CCW. This perceived inner frame tilt induces a perceived $\mathrm{CW}$ rod tilt so that the rod is set $C C W$ to appear vertical.

In conclusion, the results obtained with a central double frame arrangement replicate the effect obtained by both Zoccolotti et al. (1997) and Palmer's studies and are consistent with Rock's (1990) hierarchical organization hypothesis.

\section{EXPERIMENT 3}

Because all the experiments were performed using a circular black mask, it could be argued that this mask could have an effect on the measured illusion, by itself acting as a frame of reference.

Ebenholtz and Utrie (1983) found that an external circular annulus reduced the RFI to $23 \%$ of the usual effect. It has to be noted that they used peripheral stimuli and only a $22^{\circ}$ square frame orientation, so their study is informative on the influence that a circle had on putative visuovestibular interactions, but not on global visual effects.

To determine what effect the presence of the circle itself has on the studied illusions, we varied the salience of the circular mask by changing stimuli and background colors. We used a single frame and different orientations to measure the well-known angular function of the RFI (Wenderoth, 1974), in order to determine the effect of the mask.

\section{Method}

Subjects. Twelve new subjects ( 5 males and 7 females) were drawn from the same population as those of Experiments 1 and 2 .

Stimuli and Apparatus. The stimuli and apparatus were the same as those in Experiment 2, except for the absence of the inner inducing square.

Two different test conditions were presented: the stimuli (frame and rod) were (1) white colored on a black background and (2) black colored on a white background. The dimensions of the rod and the frame were the same as those in the previous experiment (outer frame). The mask was always black. In the condition in which both the background and the mask were black, no contrast luminance borders were present, and the aperture was not visible.

Procedure. The procedure was the same as that in Experiment 2. Three different levels of inducing square orientation $\left(11^{\circ}, 22^{\circ}\right.$, and $33^{\circ}$ ) and two levels of inducing square direction were used. The entire session lasted approximately $1 \mathrm{~h}$.

\section{Results}

The mean illusions and standard errors are shown in Figure 3. An ANOVA, with polarity and orientation conditions as repeated measures, showed an orientation effect $[F(2,22)=14.33, p<.0001]$ but no polarity effect or interaction.

When tested against zero, all the means were different from zero $(p<.01)$, except the two $22^{\circ}$ orientation effects.

\section{Discussion}

Since modifying the visibility of the black mask by changing the color of the background had no influence on the illusory effect, it is reasonable to conclude that there was no other effect except that of the inducing figure. Moreover, a circle does not act as a frame of reference when only global visual mechanisms are activated.

\section{EXPERIMENT 4}

The TI and RFI inducing stimuli differ in two main features: number of symmetry axes (see Wenderoth \& Beh, 


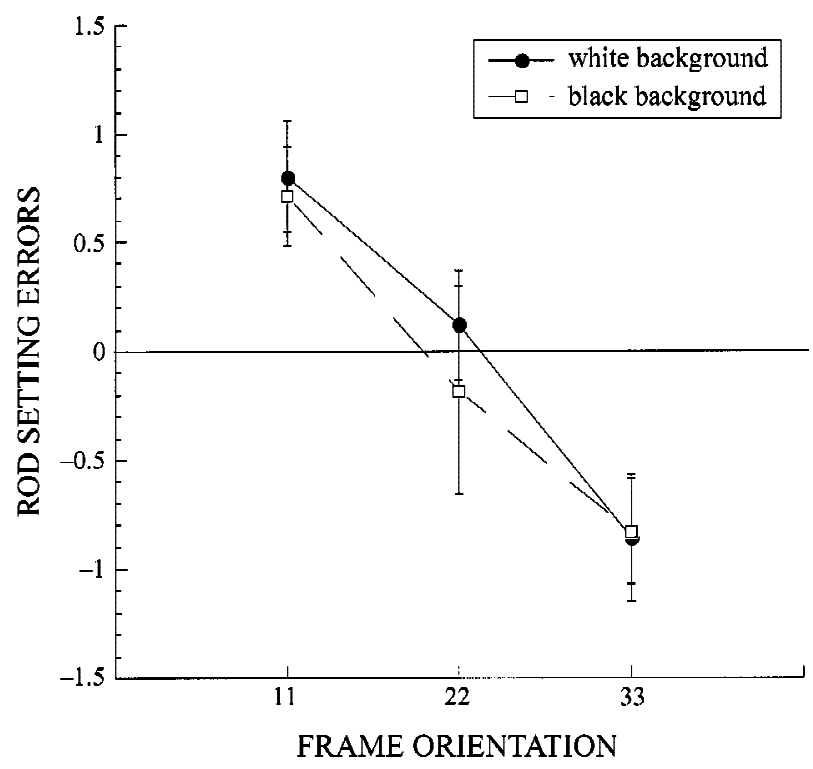

Figure 3. Mean illusion and standard errors as a function of frame tilt in the black stimuli on white background and white stimuli on black background conditions (Experiment 3).

1977) and global shape. Experiment 4 was performed to discriminate which one of these two features determines the hierarchical effect. We used a two-dimensional (2-D) TI (see Wenderoth, Johnstone, \& van der Zwan, 1989) in which each inducing annulus contained a texture composed of two orthogonal gratings (a plaid). These inducing figures have the same global shape as the TI inducers (circular) but the texture has the same number of symmetry axes as the RFI frames. ${ }^{1}$ We expected that if the hierarchical organization effect is due to the global shape, the double plaid would give results similar to the double TI, with repulsion effects to the real orientation of the inner inducing plaid. On the other hand, if it is due to the number of symmetry axes, the results would be the same as those obtained with the double RFI, with repulsion effects to the illusory perceived inner plaid orientation.

\section{Method}

Subjects. Twenty new subjects (10 males and 10 females) were drawn from the same population as those in the previous experiments.

Stimuli and Apparatus. The stimuli and apparatus were identical to those in Experiment 1, except that the inducing textures were a pair of orthogonal superimposed gratings (plaids) rather than a single grating. The outer inducing plaid was oriented so that one of the two orthogonal gratings was oriented $+11^{\circ}$ (see Figure 1C).

Procedure. The procedure was the same as that in Experiment 1. Three levels of inner inducer orientation were used: one grating at $+11^{\circ}$, the other at $-79^{\circ}$; one grating at $0^{\circ}$, and the other at $90^{\circ}$; and one grating at $-11^{\circ}$, the other at $+79^{\circ}$. The inducing and the test stimuli were of the same dimensions as the TI stimuli in Experiment 1 . The session lasted approximately $30 \mathrm{~min}$.

\section{Results}

The mean illusions and standard errors are shown in Figure 2C. Mean illusions were $+0.38^{\circ}$ when the inner frame was tilted in the opposite direction to the outer frame, $+0.86^{\circ}$ in the upright inner frame condition, and $+1.21^{\circ}$ when the inner frame was tilted in the same direction as the outer frame. The second $[t(19)=3.83, p<$ $.001]$ and last $[t(19)=5.36, p<.0001]$ of these three means were significantly different from zero.

An ANOVA with orientation condition as a repeated measure showed a significant effect $[F(2,38)=4.46, p<$ .05]. A Sheffé post hoc comparison showed that only the $\mathrm{CCW}$ and $\mathrm{CW}$ orientation effects were different from each other $(p<.05)$.

\section{Discussion}

The results obtained were compatible with neither the first nor the second prediction. Certainly, the effect was in the direction of the outer frame orientation for each inner frame condition, according to the frame-of-reference hypothesis. Thus, equating the number of symmetry axes to those of a square frame is not in itself sufficient to produce any hierarchical effect. This is probably due to the global shape of the inducing stimuli, which in the case of a circular display, give no orientation information at all. Therefore, the two-dimensionality in the plaid stimuli seems to be the feature that determines the effect according to the Zoccolotti and Palmer frame-of-reference hypothesis, in central view presentation.

These results are consistent with other studies (e.g., Poom, 2000; Wenderoth \& Johnstone, 1988a) which show that a vertical square frame (which is 2-D, like a plaid) surrounding the standard 1-D attraction TI, abolished the illusion by acting as a frame of reference.

\section{EXPERIMENT 5}

The experiments so far suggest that what determines the kind of illusory effect obtained is not only the features of the inducing stimuli, but also their position in the hierarchy. In particular, the hierarchical effect seems to occur only when the inner inducing stimulus is a square frame (or some other global shape that is capable of being perceived as tilted). At the same time, it seems that the outermost inducer acts as frame of reference only when it is 2-D.

In this last experiment, we specifically tested these two hypotheses by examining the two possible combinations of a 2-D oriented square frame as the outermost inducer (or the innermost) and a 1-D nonoriented grating annulus as the innermost inducer (or the outermost).

Thus, we used an outer annular grating with an inner square frame, predicting a hierarchical effect (a CCW error) in virtue of the presence of oriented global shape in the inner stimulus and the absence of the two-dimensionality of the outer stimulus. In the same subjects, the converse condition, an outer square frame surrounding an annular grating, was studied. We predicted that the outer 2-D stimulus would act as a frame of reference (repulsion effect, CW error), whereas the inner stimulus with no global oriented shape would have no effect. 
The target stimulus was always a rod, to avoid possible interactions of shapes and thickness between the inner and the target stimuli.

\section{Method}

Subjects. Twenty new subjects (10 males and 10 females) were drawn from the same population as those in the previous experiments.

Stimuli and Apparatus. The stimuli and apparatus were identical to those in Experiment 1, except for the presence of two different test conditions: (1) a central rod surrounded by an inner square and an outer grating (Figure 1D) and (2) a central rod surrounded by an inner grating and an outer square frame (Figure 1E). The dimensions of rod, gratings, and squares were the same as those in the previous experiments.

Procedure. The procedure was the same as that in the first experiment. Each subject was tested for each condition under three levels of inner inducer orientation: upright, tilted in the same direction as the outer frame, and tilted in the direction opposite to the outer frame. Outer inducer orientation was $+15^{\circ}$ for the grating and $+11^{\circ}$ for the square frame. Inner inducer orientation (when not $0^{\circ}$ ) was $\pm 11^{\circ}$ for the square frame and $\pm 15^{\circ}$ for the grating. The entire session lasted approximately $1 \mathrm{~h}$.

\section{Results}

The mean illusions and standard errors are shown in Figures $2 \mathrm{D}$ and $2 \mathrm{E}$. Mean illusions were $-0.32^{\circ},-0.26^{\circ}$, and $+1.67^{\circ}$ in the first configurational condition (inner square and outer grating) for the conditions in which the inner frame was tilted in same direction as the outer frame, upright, and tilted in the direction opposite the outer frame, respectively. Errors were $+0.42^{\circ},+0.88^{\circ}$, and $+0.69^{\circ}$ in the second configurational condition (inner grating and outer square) for the same inner frame orientation conditions.

An ANOVA with configurational condition and orientation condition as repeated measures showed that the orientation effect $[F(2,38)=18.30, p<.0001]$ and the configuration $\times$ orientation interaction $[F(2,38)=$ $12.31, p<.0001]$ were significant.

A Sheffé post hoc comparison showed that in the inner square frame/outer grating condition, both the opposite and the vertical orientation conditions were significantly different from the same orientation condition $(p<.0001)$, but that they did not differ from each other.

In the inner grating/outer square frame condition, the three orientations of the inner frame were not significantly different. Finally, the two configurational conditions were different from each other only at $0^{\circ}$ inner stimulus orientation $(p<.05)$.

\section{Discussion}

The results confirmed our prediction. In the inner square/outer grating condition, the results were very similar to those of Experiment 2, according to the hierarchical organization hypothesis, although the negative effects for the $0^{\circ}$ and $-11^{\circ}$ inner frame conditions were smaller. The most parsimonious explanation, given that both obtained means were negative and similar in magnitude, is that the outer grating induces an effect on the inner frame but that it is too small to produce the full hierarchical effect induced by an outer square frame.

In the inner grating/outer square condition, as in Experiment 4 , all the inner grating orientations showed an effect in the direction of the outer square frame orientation, according to a frame-of-reference hypothesis. This last experiment confirms the importance of outer frame two-dimensionality in producing a frame-of-reference effect. Moreover, it demonstrates the inability of a 1-D inner inducer without a global oriented shape to produce the inversion of effect in a hierarchical way, even if the outer stimulus does have a global oriented shape.

\section{GENERAL DISCUSSION}

First, consider the differences between orientation illusions produced by single and double inducers. In earlier experiments, several researchers used one of three kinds of single inducing stimuli: an annulus with an oriented grating texture (e.g., Wenderoth \& Johnstone, 1988b), an annulus with an oriented plaid texture (e.g., Wenderoth, van der Zwan, \& Williams, 1993), or a tilted square frame (e.g., Wenderoth, 1974). The resulting illusory orientation effects on a test stimulus-either a vertical grating or a single rod-varied in amplitude and direction with the angular distance of either the real or the virtual symmetry axes of the inducer from the objective vertical. This illusory effect was qualitatively similar for each inducing stimulus. However, our experiments have demonstrated that double concentric inducers produce qualitatively different phenomena, depending on the type of inducing stimuli.

Two main features determine the kind of effect that occurs with multiple inducers: the presence/absence of a global shape capable of signaling a direction and the number of symmetry axes of the inducing figure. Also, the closeness/distance from the test stimulus in the hierarchy is relevant to the efficacy of these features in inducing either a hierarchical effect or a frame-of-reference effect.

A 2-D stimulus, such as a square or a plaid, is able to induce a direct effect on the test stimulus when it is the outermost of the two inducers, regardless of the orientation direction of the inner inducer (Experiments 4 and 5). A similar result was obtained in central vision when a TI display was surrounded by an upright square frame (Wenderoth \& Johnstone, 1988a; Wenderoth \& van der Zwan, 1989).

A 1-D stimulus is not able to induce the same direct effect when it is the outermost of the two inducers. Experiment 1 shows that when the outer inducer is a grating annulus, the bulk of the illusory effect is attributable to the inner inducer.

The contrast between 1-D and 2-D TI results (Experiments 1 and 4) led us to suggest that only a 2-D, but not a 1-D, stimulus can act as a frame of reference.

The double square frame used in Experiment 2 and the inner-square/outer-grating inducing figure of Experi- 
ment 5 were the only two conditions to produce an effect from the outermost to the innermost inducer, affecting only the stimulus closest to it. Since the square frame was the only figure we used that had a global oriented shape, it is likely that this feature is required in the inner inducer in order to produce the hierarchical organization effect.

The difference between a 1-D (Experiment 5) and a 2-D (Experiment 2) external frame in making the inner square frame appear tilted could be evidence that the two described effects can be interactive.

Taken together, the results of Experiments 2, 4, and 5 suggest that hierarchical organization and frame of reference are not opponent phenomena, given that a hierarchical organization effect can be induced by the same outermost stimulus that can induce a frame-of-reference effect (e.g., an outer square frame). Nevertheless, the inducing stimulus closest to the target must have a global oriented shape to prime a hierarchical illusory effect.

Our results corroborate neither Rock's (1990) theory about the importance of size to induce one or the other effect, nor Zoccolotti et al.'s (1997) hypothesis that hierarchical organization is due to global mechanisms and the frame of reference is a result of visuo-vestibular interactions. We found that both effects are present in a small display in which we believe that only global mechanisms are present.

If we consider together the experiments carried out with small and large displays, we could speculate that both global mechanisms and visuo-vestibularinteractions can induce both kinds of effects but that the main features to trigger them are different. A circle can act as frame of reference in a peripheral display (Ebenholtz \& Utrie, 1983), but not in a central display (Experiment 3 ).

An inner square frame is able to isolate the test stimulus from the effect of an outer frame of reference in a central display (Experiment 2), but not in peripheral view (DiLorenzo \& Rock, 1982; Zoccolotti et al., 1997).

Although we have chosen to confine accounts of our data to global psychophysical hypotheses, we are aware that several global, or long-range, effects have recently been given explanations in terms of long-range horizontal connections in the V1 cortex (e.g., Brincat \& Westheimer, 2000; Wehrhahn, Li, \& Westheimer, 1996; Westheimer, Brincat, \& Wehrhahn, 1999). It is entirely plausible that such connections play an integral part in the phenomena we have reported, although it is beyond the scope of this paper to elaborate on that possibility.

In conclusion, the global visual mechanism responsible for illusory effects of orientation in central view presentation displays induce either a frame-of-reference or a hierarchical organization effect, depending on the precise features of the inducing stimuli chosen. In a double frame condition, a frame-of-reference effect arises from a 2-D outermost stimulus, whereas a hierarchical organization effect is exhibited only in presence of a stimulus with an oriented global shape that is the closest to the target. A speculative reason could be that the outermost oriented stimulus can be anything acting as a spatial axes surrogate (therefore, 2-D), whereas the inner stimulus must be an "object" that contains the target. An annulus cannot be encoded as a tilted object, regardless of the texture (e.g., grating or plaid) it contains, whereas a square can be encoded as an object that can vary in tilt and so can have an orientation shift induced upon it by a surrounding tilted object.

\section{REFERENCES}

Antonucci, G., Fanzon, D., Spinelli, D., \& Zoccolotti, P. (1995). Visual factors affecting the rod and frame illusion: Role of gap size and frame components. Perception, 24, 1119-1130.

Blakemore, C. B., Carpenter, R. H. S., \& Georgeson, M. A. (1970). Lateral inhibition between orientation detectors in the human visual system. Nature, 228, 37-39.

BRINCAT, S. L., \& WeSTHEIMER, G. (2000). Integration of foveal orientation signals: Distinct local and long-range domains. Journal of Neurophysiology, 83, 1900-1911.

DAINI, R. (1998). Effect of shape on verticality judgements with single and double frameworks. Perception, 27 (Suppl.), 87.

DiLorenzo, J. R. \& Rock, I. (1982). The rod-and-frame effect as a function of the righting of the frame. Journal of Experimental Psychology: Human Perception \& Performance, 8, 536-546.

Ebenholtz, S. M. (1985). Depth separation fails to modulate the orientation-inhibitioneffect. Perception \& Psychophysics, 37, 533-535.

Ebenholtz, S. M., \& Benzschawel, T. L. (1977). The rod and frame effect and induced head tilt as a function of observation distance. Perception \& Psychophysics, 22, 491-496.

Ebenholtz, S. M., \& Utrie, J. W., JR. (1982). Inhibition of the rodand-frame effect by circular contours. Perception \& Psychophysics, 32, 199-200.

Ebenholtz, S. M., \& Utrie, J. W., Jr. (1983). Peripheral circular contours inhibit the visual orientation control system. Aviation, Space, \& Environmental Medicine, 54, 343-346.

Goodenough,D. R., Sigman, E., Oltman, P. K., Rosso, J., \& Mertz, H (1979). Eye torsion in response to a tilted visual stimulus. Vision Research, 19, 1177-1179.

Howard, I. P. (1982). Human visual orientation. New York: Wiley.

Poом, L. (2000). Inter-attribute tilt effects and orientation analysis in the visual brain. Vision Research, 40, 2711-2722.

Rock, I. (1990). The frame of reference. In I. Rock (Ed.), The legacy of Solomon Asch (pp. 243-268). Hillsdale, NJ: Erlbaum.

Spinelli, D., Antonucci, G., Daini, R., Fanzon, D., \& Zoccolotti, P. (1995). Modulation of the rod-and-frame illusion by additional external stimuli. Perception, 24, 1105-1118.

Spinelli, D., Antonucci, G., Daini, R., Martelli, M. L., \& ZocColotTI, P. (1999). Hierarchical organization in perception of orientation. Perception, 28, 965-980.

Spinelli, D., Antonucci, G., DAini, R, \& Zoccolotti, P. (1995). Local and global visual mechanisms underlying individual differences in the rod-and-frame illusion. Perception \& Psychophysics, 57, 915-920.

Tolmurst, D. J., \& Thompson, P. G. (1975). Orientation illusions and after-effects: Inhibition between channels. Vision Research, 15, 967 972.

VAN DER Zwan, R., \& Wenderoth, P. (1995). Mechanisms of purely subjective contour tilt aftereffects. Vision Research, 35, 2547-2557.

Virsu, V., \& TASKinen, H. (1975). Central inhibitory interactions in human vision. Experimental Brain Research, 23, 65-74.

WAllace, G. K. (1969). The critical distance of interaction in the Zöllner illusion. Perception \& Psychophysics, 5, 261-264.

Wehrhahn, C., LI, W., \& Westheimer, G. (1996). Patterns that impair discrimination of line orientation in human vision. Perception, 25, 1053-1064.

Wenderoth, P. (1974). The distinction between the rod-and-frame illusion and the rod-and-frame test. Perception, 3, 205-212.

WENDEROTH, P. (1997). The role of implicit axes of bilateral symmetry in orientation processing. Australian Journal of Psychology, 49, 176-181. 
Wenderoth, P., \& Beh, H. (1977). Component analysis of orientation illusions. Perception, 6, 57-75.

Wenderoth, P., \& Johnstone, S. (1987). Possible neural substrates for orientation analysis and perception. Perception, 16, 693-709.

Wenderoth, P., \& Johnstone, S. (1988a). The differential effects of brief exposures and surrounding contours on direct and indirect tilt illusions. Perception, 17, 165-176.

Wenderoth, P., \& Johnstone, S. (1988b). The different mechanisms of the direct and indirect tilt illusions. Vision Research, 28, 301-312.

Wenderoth, P., Johnstone, S., \& VAN Der Zwan, R. (1989). Two dimensional tilt illusions induced by orthogonal plaid patterns: Effects of plaid motion, orientation, spatial separation and spatial frequency. Perception, 18, 25-38.

Wenderoth, P., \& VAN DER ZWAN, R. (1989). The effects of exposure duration and surrounding frames on direct and indirect tilt aftereffects and illusions. Perception \& Psychophysics, 46, 338-344.

Wenderoth, P., \& VAN DER ZWAN, R. (1991). Local and global mechanisms of one- and two-dimensional orientation illusions. Perception \& Psychophysics, 50, 321-332.

Wenderoth, P., van der Zwan, R., \& Williams, M. (1993). Direct evidence for competition between local and global mechanisms of two-dimensional orientation illusions. Perception, 22, 273-286.

Westheimer, G., Brincat, S., \& Wehrhahn, C. (1999). Contrast de- pendency of foveal spatial functions: Orientation, vernier separation, blur and displacement discrimination and the tilt and Poggendorff illusions. Vision Research, 39, 1631-1639.

WetheriLl, G., \& Levitt, H. (1965). Sequential estimation of points on a psychometric function. British Journal of Mathematical \& Statistical Psychology, 18, 1-10.

Zoccolotti, P., Antonucci, G., Daini, R., Martelli, M. L., \& SPINELLI, D. (1997). Frame-of-reference and hierarchical-organization effects in the rod-and-frame illusion. Perception, 26, 1485-1494.

Zoccolotti, P., Antonucci, G., \& Spinelli, D. (1993). The gap between rod and frame influences the rod-and-frame effect with small and large inducing displays. Perception \& Psychophysics, 54, 14-19.

\section{NOTE}

1. A square frame and a grating also differ in texture and thickness, with the plaid being more similar to the grating than to the square. Nevertheless, these two features cannot account for the presence of the hierarchical organization effect in the double RFI but its absence in the double TI (see Daini, 1998).

(Manuscript received February 25, 2002; revision accepted for publication January 31, 2003.) 